\title{
The moduli b-divisor of an lc-trivial fibration
}

\author{
Florin Ambro
}

\begin{abstract}
We study positivity properties of the moduli (b-)divisor associated to a relative log pair $(X, B) / Y$ with relatively trivial log canonical class.
\end{abstract}

\section{Introduction}

In this paper we continue the study of lc-trivial fibrations $(X, B) / Y$, that is, roughly, relative log pairs with relatively trivial log canonical class $K+B$ (see [Amb02] for an introduction). This type of fibration is expected to play a key role in inductive arguments in the (Log) Minimal Model Program.

We briefly explain the inductive idea on an example, which is the prototype of lc-trivial fibrations. Consider a fibration of projective manifolds $f: X \rightarrow Y$ such that $K_{X}=f^{*} D$, where $K_{X}$ is the canonical divisor of $X$ and $D$ is a Cartier divisor on $Y$. It is clear that some of the basic invariants of $X$, such as its Kodaira dimension or its plurigenera, are encoded by the divisor $D$, which is defined on a variety of dimension strictly less than that of $X$. In the ideal case when $D$ is the canonical divisor of $Y$, results in the classification theory of $Y$ imply analogous results in the classification theory of $X$ (e.g. the base point freeness of the pluricanonical linear systems, or the finite generation of the canonical ring). In general, $D$ is different from the canonical divisor of $Y$ due to the existence of singular fibers of $f$ and the variation of the generic fiber of $f$ in its moduli (in fact, $D=K_{Y}$ if and only if $f$ is a product after a finite étale base change, by Theorem 4.7). What is always true is that $D$ is the $\log$ canonical divisor of a logarithmic variety (see Theorem 0.2 ), and the inductive argument sketched above is valid in the larger category of logarithmic varieties.

Given an lc-trivial fibration $f:(X, B) \rightarrow Y$, there exists a canonical decomposition of Kodaira type

$$
K+B \sim_{\mathbb{Q}} f^{*}\left(K_{Y}+B_{Y}+M_{Y}\right),
$$

where $B_{Y}$ and $M_{Y}$ are $\mathbb{Q}$-divisors on $Y$, called the discriminant and moduli $\mathbb{Q}$-divisors (Kawamata [Kaw97, Kaw98]). The discriminant measures the singularities of the log pair $(X, B)$ over codimension-1 points of $Y$, whereas the moduli $\mathbb{Q}$-divisor is expected to define the rational map from $Y$ to the moduli space of the generic fiber. As explained in [Amb02], the following two properties are desirable for applications: inversion of adjunction and (effective) semi-ampleness. Inversion of adjunction (or, equivalently, Shokurov's BP Conjecture [Sho03]) was established in [Amb02]: the log pairs $(X, B)$ and $\left(Y, B_{Y}\right)$ have the same type of singularities if $Y$ is sufficiently high in its birational class. As for the moduli part, it is known that $M_{Y}$ is numerically effective (nef) if $Y$ is sufficiently high in its birational class [Kaw98]. Semi-ampleness predicts that in fact the linear system $\left|k M_{Y}\right|$ is free of base points if $k$ is large and divisible and $Y$ is sufficiently high in its birational class.

The main results of this paper are two partial answers to the semi-ampleness of the moduli part: (a) if $M_{Y}$ is numerically trivial and $Y$ is sufficiently high in its birational class, then $M_{Y}$ is a torsion

Received 20 September 2003, accepted in final form 26 February 2004, published online 10 February 2005. 2000 Mathematics Subject Classification 14J10, 14N30 (primary), 14E30 (secondary).

Keywords: lc-trivial fibrations, adjunction formulas, log varieties, variation of Hodge structure.

This journal is (C) Foundation Compositio Mathematica 2005. 


\section{F. AmBro}

Q-divisor (Theorem 3.5); (b) if the horizontal part of $B$ is effective and $Y$ is sufficiently high in its birational class, then there exists a contraction $h: Y \rightarrow Z$ to a projective variety $Z$ and a nef and big $\mathbb{Q}$-divisor $A$ on $Z$ such that $M_{Y} \sim_{\mathbb{Q}} h^{*} A$ (Theorem 3.3).

One application of Theorem 3.3 is a logarithmic version of a result of Kawamata [Kaw85] (the Abundance Conjecture and Ueno's K Conjecture for minimal models with numerically trivial canonical class), as follows.

Theorem 0.1. Let $(X, B)$ be a projective log variety with Kawamata log terminal singularities such that $K+B$ is numerically trivial. Then the following hold.

(1) There exists a positive integer $b$ such that $b(K+B) \sim 0$.

(2) The Albanese map $X \rightarrow \operatorname{Alb}(X)$ is a surjective morphism, with connected fibers. Furthermore, there exist a finite étale covering $A^{\prime} \rightarrow \operatorname{Alb}(X)$, a projective $\log$ variety $\left(F, B_{F}\right)$, and an isomorphism over $A^{\prime}$ such that

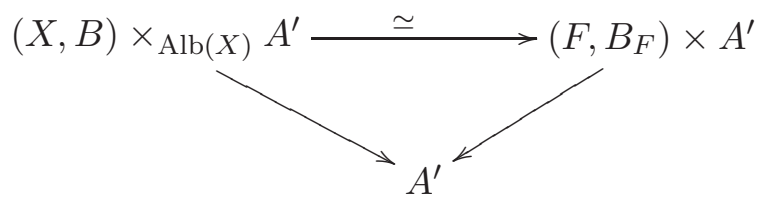

It is interesting to compare this with results of Campana, Demailly, Peternell, Schneider, Serrano, and Zhang [DPS93, Zha96, PS98, CPZ03] on the structure of the Albanese map of projective manifolds with nef anticanonical class. By Theorem 0.1, the Albanese map of a nonsingular projective variety with semi-ample anticanonical class $-K$ is an étale fiber bundle.

Another application of Theorem 3.3 is a generalization of a result of Nakayama [Nak88] (see also Fujita [Fuj86]), as follows.

Theorem 0.2. Let $(X, B)$ be a projective log variety with Kawamata log terminal singularities, let $f: X \rightarrow Y$ be a contraction to a proper normal variety $Y$, and let $\omega$ be a $\mathbb{Q}$-Cartier divisor on $Y$ such that

$$
K+B \sim \mathbb{Q} f^{*} \omega .
$$

Then there exists a $\mathbb{Q}$-Weil divisor $B_{Y}$ such that $\left(Y, B_{Y}\right)$ is a log variety with Kawamata log terminal singularities and $\omega \sim \sim_{\mathbb{Q}} K_{Y}+B_{Y}$.

Another application is the logarithmic version of the main result in [Amb03]: modulo the Log Minimal Model Program and the Log Abundance Conjecture for smaller dimensional varieties, the Log Abundance Conjecture is reduced to the case of log minimal models of maximal nef dimension (Theorem 4.3).

The techniques we use are due to Fujita [Fuj78a, Fuj78b], Viehweg [Vie83a, Vie83b] and Kawamata [Kaw83, Kaw85]. In fact, Theorem 3.3 can be deduced from [Kaw85, Theorem 1.1] in the case in which the generic fiber $X_{\eta}$ has canonical singularities and $B_{\eta}=0$ (see Fujino and Mori [FM00, Fuj03], or [Amb03]). Thus, we simply extend their methods to deal with the case of varieties with boundary.

\section{Preliminary}

Since we use transcendental methods, the base field is assumed to be the field of complex numbers $k=\mathbb{C}$. However, the main results extend to the case when $k$ is an algebraically closed field of characteristic 0, by Lefschetz's principle.

We use the same notation and terminology as in [Amb02]. Recall that a log variety with Kawamata $\log$ terminal singularities is a normal variety $X$ endowed with an effective $\mathbb{Q}$-Weil 


\section{THE MODULI B-DIVISOR}

divisor $B$ satisfying the following two properties: (a) some integer multiple of the log canonical class $K+B$ is a Cartier divisor; (b) for any resolution of singularities $\mu: Y \rightarrow X$, the $\mathbb{Q}$-Weil divisor $K_{Y}-\mu^{*}(K+B)$ has coefficients less than 1 (the same top rational differential form $\omega \in \wedge^{\operatorname{dim}(X)} \Omega_{k(X) / k}^{1}$ is used to define the canonical class $K_{Y}=(\omega)_{Y}$ of $Y$, for every birational model $Y$ of $X$ ).

Throughout this paper, we only consider complex-analytic spaces which are associated to complex algebraic varieties (a general definition can be found in [Hir64, pp. 119-120]). To fix the notation, let $X \stackrel{\pi}{\rightarrow} S$ be a morphism of complex-analytic spaces. The tangent sheaf $T_{X / S}=\left(\Omega_{X / S}^{1}\right)^{\vee}$ is the dual of the sheaf of relative Kähler differentials $\Omega_{X / S}^{1}$. If $X$ is smooth and $E$ is a divisor with simple normal crossings on $X, \Omega_{X}^{1}\langle E\rangle$ is the locally free sheaf of differentials with logarithmic poles along $E, \Omega_{X / S}^{1}\langle E\rangle$ is defined by the exact sequence

$$
\pi^{*} \Omega_{S}^{1} \rightarrow \Omega_{X}^{1}\langle E\rangle \rightarrow \Omega_{X / S}^{1}\langle E\rangle \rightarrow 0,
$$

and the logarithmic tangent sheaf $T_{X / S}\langle-E\rangle$ is defined as the dual of $\Omega_{X / S}^{1}\langle E\rangle$ (it coincides with the sheaf of derivations of $X$ which preserve $\mathcal{I}_{E}$ ). For the deformation theory of a complex-analytic space, or that of a smooth complex-analytic space endowed with a simple normal crossings divisor, we refer the reader to [GK64, Gra74, Kaw78].

For the rest of this section, we collect some results of Kawamata [Kaw83, Kaw85], Kollár [Kol87] and Viehweg [Vie83a, Vie83b], with minor modifications.

\subsection{Equivariant resolutions}

Let $X$ be a complex-analytic space which is countable at infinity and let $Z \supset \operatorname{Sing}(X)$ be a closed complex subspace. By Hironaka [Hir77], there exists a proper morphism $\mu: X^{\prime} \rightarrow X$ having the following properties.

(1) $X^{\prime}$ is smooth and $\mu$ induces an isomorphism $X^{\prime} \backslash \mu^{-1}(Z) \rightarrow X \backslash Z$.

(2) $\mu^{-1}(Z)$ is a divisor with normal crossings support.

(3) If $U, V$ are open subsets of $X$ and $\alpha: U \rightarrow V$ is an isomorphism such that $\alpha\left(\left.Z\right|_{U}\right)=\left.Z\right|_{V}$, then there exists a unique isomorphism $\alpha^{\prime}$ making the following diagram commutative:

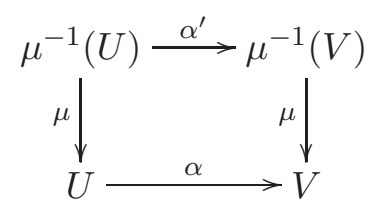

(4) Let $\mathcal{P}$ be the pseudo-group of all the local isomorphisms $\alpha$ as in (3). Then $\mu$ is obtained as the composition of blowing-ups with closed smooth centers which are invariant under the natural liftings of $\mathcal{P}$.

We will say that $\mu$ is an equivariant resolution of $X$ with respect to $Z$.

Lemma 1.1. Let $X$ be a normal variety, let $R$ be a reduced Weil divisor on $X$ (possibly zero), and let $X \rightarrow S$ be a morphism. Let $\mu: X^{\prime} \rightarrow X$ be an equivariant resolution of $X$ with respect to $\operatorname{Sing}(X) \cup R$, and let $E$ be a normal crossings divisor on $X^{\prime}$ such that $R \subseteq \mu_{*} E$. Then

$$
\mu_{*} T_{X^{\prime} / S}\langle-E\rangle=\left(\mu_{*} \Omega_{X^{\prime} / S}^{1}\langle E\rangle\right)^{\vee} \subseteq T_{X / S} .
$$

Proof. Since $X$ is normal and $\mu$ is birational, we have inclusions

$$
\mu_{*} T_{X^{\prime} / S}\langle-E\rangle \subseteq\left(\mu_{*} \Omega_{X^{\prime} / S}^{1}\langle E\rangle\right)^{\vee} \subseteq T_{X / S}
$$




\section{F. AmBro}

Fix a point $x \in X$ and let $\mathfrak{a} \in H^{0}\left(U,\left(\mu_{*} \Omega_{X^{\prime} / S}^{1}\langle E\rangle\right)^{\vee}\right) \subset H^{0}\left(U, T_{X / S}\right)$ be a vector field on an analytic neighborhood $U$ of $x$. There exists (see [GK64]) a holomorphic one-parameter family $\Phi:\{s \in \mathbb{C} ;|s|<\epsilon\} \times U^{\prime} \rightarrow U$ satisfying the following properties:

(a) $\Phi\left(s_{1}, \Phi\left(s_{2}, x\right)\right)=\Phi\left(s_{1}+s_{2}, x\right)$ for $\left|s_{1}\right|,\left|s_{2}\right|,\left|s_{1}+s_{2}\right|<\epsilon$;

(b) $\Phi(0, x)=x$ for $x \in U^{\prime}$;

(c) $\mathfrak{a}_{x}(d f)=\left.\frac{d}{d s} f(\Phi(s, x))\right|_{s=0}$ for $x \in U^{\prime}$ and $f \in \mathcal{O}_{X, x}$.

The local isomorphisms $\Phi_{s}$ preserve the singular locus of $X$. They also preserve $R$ on a big open subset of $X$, since $\mu$ is birational and $R \subseteq \mu_{*} E$. Therefore each $\Phi_{s}$ preserves $\operatorname{Sing}(X) \cup R$, so $\Phi$ lifts to a one-parameter family in an analytic neighborhood of $\pi^{-1}(x)$. The corresponding vector field $\tilde{\mathfrak{a}}$ is tangent to the exceptional locus of $\mu$ and to the components of $E$. Then $\tilde{\mathfrak{a}}$ is a section of $T_{X^{\prime} / S}\langle-E\rangle$ which lifts $\mathfrak{a}$, hence $\mathfrak{a} \in \mu_{*} T_{X^{\prime} / S}\langle-E\rangle$.

Lemma 1.2. Let $\mu: Y \rightarrow X$ be a birational morphism, let $B$ be a sheaf on $X$, and let $A$ be a torsion-free sheaf on $Y$. Then we have an exact sequence

$$
0 \rightarrow \operatorname{Ext}_{X}^{1}\left(B, \mu_{*} A\right) \rightarrow \operatorname{Ext}_{Y}^{1}\left(\mu^{*} B, A\right) \rightarrow \operatorname{Hom}_{X}\left(B, R^{1} \mu_{*} A\right) .
$$

Proof. If we set $\operatorname{Hom}_{\mu}(B, A)=\operatorname{Hom}_{Y}\left(\mu^{*} B, A\right)=\operatorname{Hom}_{X}\left(B, \mu_{*} A\right)$, we have two spectral sequences [Ran89]:

$$
\begin{aligned}
& E_{2}^{p, q}=\operatorname{Ext}_{X}^{p}\left(B, R^{q} \mu_{*} A\right) \Longrightarrow \operatorname{Ext}_{\mu}^{p+q}(B, A), \\
& { }^{\prime} E_{2}^{p, q}=\operatorname{Ext}_{Y}^{p}\left(L_{q} \mu^{*} B, A\right) \Longrightarrow \operatorname{Ext}_{\mu}^{p+q}(B, A) .
\end{aligned}
$$

The five-term exact sequence of edge homomorphisms of the first spectral sequence is (denote $\left.H^{i}=\operatorname{Ext}_{\mu}^{i}(B, A)\right)$

$$
0 \rightarrow \operatorname{Ext}_{X}^{1}\left(B, \mu_{*} A\right) \rightarrow H^{1} \rightarrow \operatorname{Hom}_{X}\left(B, R^{1} \mu_{*} A\right) \rightarrow \operatorname{Ext}_{X}^{2}\left(B, \mu_{*} A\right) \rightarrow H^{2} .
$$

Since $\mu$ is birational, $L_{1} \mu^{*} B$ is a torsion sheaf on $Y$. Since $A$ is torsion-free, we obtain ${ }^{\prime 0,1}=$ $\operatorname{Hom}_{Y}\left(L_{1} \mu^{*} B, A\right)=0$. Therefore the second spectral sequence induces an isomorphism $\operatorname{Ext}_{Y}^{1}\left(\mu^{*} B, A\right) \stackrel{\sim}{\longrightarrow} H^{1}$.

\subsection{The covering trick}

Let $f: X \rightarrow S$ be a smooth projective morphism of nonsingular varieties, and let $T$ be a $\mathbb{Q}$-divisor on $X$ such that $T \sim \mathbb{Q} 0$ and such that the fractional part of $T$ has normal crossings support relative to $f$. Let $\varphi$ be a rational function on $X$ such that $(\varphi)+m T=0$ (and $m$ is minimal with this property). Let $\pi: \tilde{X} \rightarrow X$ be the normalization of $X$ in $k(X)(\sqrt[m]{\varphi})$, and let $\nu: V \rightarrow \tilde{X}$ be an equivariant resolution with respect to the singular locus of $\tilde{X}$. We assume that the morphism $h: V \rightarrow S$ is smooth and $R^{2} h_{*} \mathbb{Z}_{V}$ has a global section inducing a polarization on each fiber of $h$.

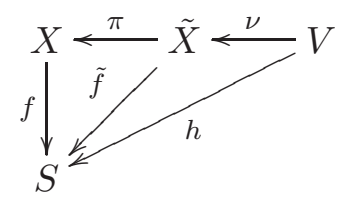

We have an eigensheaf decomposition

$$
h_{*} K_{V / S}=\tilde{f}_{*} K_{\tilde{X} / S}=\bigoplus_{i=0}^{m-1} f_{*} \mathcal{O}_{X}\left(\left\lceil K_{X / S}+i T\right\rceil\right) .
$$

In particular, we obtain locally-free sheaves on $S$ :

$$
\mathcal{F}^{(i)}:=f_{*} \mathcal{O}_{X}\left(\left\lceil K_{X / S}+i T\right\rceil\right) .
$$




\section{The MOdULi B-DIVISOR}

The variation of polarized Hodge structure $\left(R^{d} h_{*} \mathbb{C}_{V}\right)_{\text {prim }} \otimes \mathcal{O}_{S}$ induces semipositive Hermitian metrics on each $\mathcal{F}^{(i)}$. Let

$$
\sigma_{s}^{i}: T_{S, s} \rightarrow \operatorname{Hom}\left(H^{0}\left(X_{s}, \mathcal{F}_{s}^{(i)}\right), \operatorname{Ext}_{X_{s}}^{1}\left(\Omega_{X_{s}}^{1}\left\langle E_{s}\right\rangle, \mathcal{F}_{s}^{(i)}\right)\right)
$$

be the map induced by cup product with the Kodaira-Spencer class

$$
\kappa_{s}: T_{S, s} \rightarrow \operatorname{Ext}_{X_{s}}^{1}\left(\Omega_{X_{s}}^{1}\left\langle E_{s}\right\rangle, \mathcal{O}_{X_{s}}\right),
$$

where $E$ is the support of the fractional part of $T$.

Proposition 1.3. The following properties hold.

(i) Viewed as a subbundle of the flat vector bundle $\left(R^{d} h_{*} \mathbb{C}_{V}\right)_{\text {prim }} \otimes \mathcal{O}_{S}$, the second fundamental forms of $\mathcal{F}^{(i)}$ and $h_{*} \omega_{V / S}$ are represented by $\sigma^{i}$ and $\bigoplus_{i=0}^{m-1} \sigma^{i}$, respectively.

(ii) The Hermitian vector bundle $\mathcal{F}^{(i)}$ is Griffiths semipositive definite, with curvature

$$
\Theta_{i}=-\left(\sigma^{i}\right)^{*} \wedge \sigma^{i}
$$

where $\left(\sigma^{i}\right)^{*}(v)$ is the adjoint of $\sigma^{i}(\bar{v})$ with respect to the induced Hodge metrics. The curvature $\operatorname{trace}\left(\Theta_{i}\right)$ of $\operatorname{det}\left(\mathcal{F}^{(i)}\right)$ is semipositive, and $\operatorname{Ker}\left(\sigma_{s}^{i}\right) \subset T_{S, s}$ consists of the tangent directions along which trace $\left(\Theta_{i}\right)$ is not positive definite at $s$.

(iii) The maps $\sigma_{V_{s}}, \sigma_{\tilde{X}_{s}}, \bigoplus_{i=0}^{m-1} \sigma_{s}^{i}$ and $\kappa_{V_{s}}, \kappa_{\tilde{X}_{s}}, \kappa_{s}$ have the same kernel, respectively.

(iv) Assume $S \subset \bar{S}$ is a nonsingular compactification of $S$ such that $\bar{S} \backslash S$ is a simple normal crossings divisor and $R^{d} h_{*} \mathbb{C}_{V}$ has unipotent local monodromies along $\bar{S} \backslash S$. Let $\overline{\mathcal{F}}^{(i)}$ be the Schmid extension of $\mathcal{F}^{(i)}$. Then $\operatorname{det}\left(\overline{\mathcal{F}}^{(i)}\right)$ is a nef invertible sheaf on $\bar{S}$, and

$$
\operatorname{det}\left(\overline{\mathcal{F}}^{(i)}\right)^{\operatorname{dim}(S)}=\int_{S}\left(\frac{\sqrt{-1}}{2 \pi} \operatorname{trace}\left(\Theta_{i}\right)\right)^{\operatorname{dim}(S)} .
$$

Proof. (i) We may shrink $S$, so that $V_{s} \rightarrow \tilde{X}_{s}$ is a resolution of singularities for every $s \in S$. We have an inclusion of Hodge structures $H^{d}\left(\tilde{X}_{s}, \mathbb{C}\right) \subset H^{d}\left(V_{s}, \mathbb{C}\right)$ and $H^{d, 0}\left(\tilde{X}_{s}\right)=H^{d, 0}\left(V_{s}\right)$, since $\tilde{X}_{s}$ has rational singularities (see [Ste77]). Therefore we have the commutative diagram

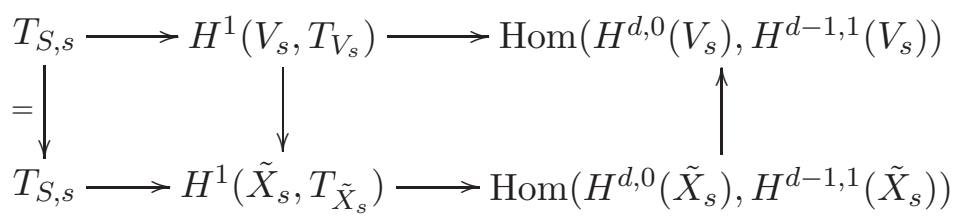

where the middle arrow is the tangent map of the blow-down transformation of deformation functors [Kaw85]. If we identify [Vie83b]

$$
H^{1}\left(X_{s}, T_{X_{s}}\left\langle-E_{s}\right\rangle\right)=H^{1}\left(\tilde{X}_{s}, T_{\tilde{X}_{s}}\right)^{G},
$$

we also have a commutative diagram:

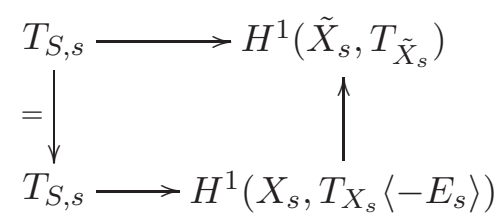

Therefore the cup product with the Kodaira-Spencer class preserves the eigenspaces, so that the infinitesimal period map

$$
\sigma_{V_{s}}: T_{S, s} \rightarrow \operatorname{Hom}\left(H^{d, 0}\left(V_{s}\right), H^{d-1,1}\left(V_{s}\right)\right)
$$




\section{F. AmBro}

admits the decomposition $\sigma_{V_{s}}=\bigoplus_{i=0}^{m-1} \sigma_{s}^{i}$. This is the same as the action of the Gauss-Manin connection on $\left(R^{d} h_{*} \mathbb{C}_{V}\right)_{\text {prim }} \otimes \mathcal{O}_{S}$, hence $\sigma_{s}^{i}$ is the second fundamental form of $\mathcal{F}^{(i)}$ in $\left(R^{d} h_{*} \mathbb{C}_{V}\right)_{\text {prim }} \otimes \mathcal{O}_{S}$.

(ii) See [Gri70].

(iii) Consider the infinitesimal period maps

$$
\begin{aligned}
\sigma_{V_{s}}: T_{S, s} & \rightarrow \operatorname{Hom}\left(H^{d, 0}\left(V_{s}\right), H^{d-1,1}\left(V_{s}\right)\right), \\
\sigma_{\tilde{X}_{s}}: T_{S, s} & \rightarrow \operatorname{Hom}\left(H^{d, 0}\left(\tilde{X}_{s}\right), H^{d-1,1}\left(\tilde{X}_{s}\right)\right), \\
\sigma_{s}^{i}: T_{S, s} & \rightarrow \operatorname{Hom}\left(H^{0}\left(X_{s}, \mathcal{F}_{s}^{(i)}\right), \operatorname{Ext}_{X_{s}}^{1}\left(\Omega_{X_{s}}^{1}\left\langle E_{s}\right\rangle, \mathcal{F}_{s}^{(i)}\right)\right)
\end{aligned}
$$

and the Kodaira-Spencer deformation classes

$$
\begin{aligned}
\kappa_{V_{s}}: T_{S, s} & \rightarrow H^{1}\left(V_{s}, T_{V_{s}}\right), \\
\kappa_{\tilde{X}_{s}}: T_{S, s} & \rightarrow H^{1}\left(\tilde{X}_{s}, T_{\tilde{X}_{s}}\right), \\
\kappa_{s}: T_{S, s} & \rightarrow H^{1}\left(X_{s}, T_{X_{s}}\left\langle-E_{s}\right\rangle\right) .
\end{aligned}
$$

The proof of (i) gives that $\sigma_{V_{s}}, \sigma_{\tilde{X}_{s}}, \bigoplus_{i=0}^{m-1} \sigma_{s}^{i}$ and $\kappa_{\tilde{X}_{s}}, \kappa_{s}$ have the same kernel, respectively. Since $\nu$ is equivariant, $\kappa_{V_{s}}$ and $\kappa_{\tilde{X}_{s}}$ have the same kernel by [Kaw85, Lemma 6.2].

(iv) This follows from [Kaw83] and [Kol87].

\section{The period map}

Throughout this section, we fix the following setup:

(a) $(X, B)$ is a log variety with Kawamata log terminal singularities, such that $K+B \sim_{\mathbb{Q}} 0$;

(b) $f: X \rightarrow S$ is a projective contraction to a nonsingular algebraic variety $S$;

(c) $\mu: Y \rightarrow X$ is a resolution of singularities, $K_{Y}+B_{Y}=\mu^{*}(K+B)$ is the log pullback, and $E$ is the support of the fractional part of $B_{Y}$. We assume that $E$ has simple normal crossings support and $\mu_{*} T_{Y}\langle-E\rangle$ is a reflexive sheaf (such a resolution exists by Lemma 1.1);

(d) the family $(Y, E) \rightarrow S$ has relative simple normal crossings over an open subset of $S$. Let $\kappa_{s}: T_{S, s} \rightarrow H^{1}\left(Y_{s}, T_{Y_{s}}\left\langle-E_{s}\right\rangle\right)$ be the induced Kodaira-Spencer class.

We are only interested in the induced $\log$ variety $\left(X_{\eta}, B_{\eta}\right)$ defined over $k(S)$. Hence, we will shrink $S$ to a Zariski open subset without further notice.

Proposition 2.1. Let $b$ be the minimal positive integer such that $b(K+B) \sim 0$ over the general point of $S$, and choose a rational function $\varphi$ with $b(K+B)=(\varphi)$. Let $\pi: \tilde{Y} \rightarrow Y$ be the normalization of $Y$ in $k(Y)(\sqrt[b]{\varphi})$, and let $\nu: V \rightarrow \tilde{Y}$ be an equivariant resolution with respect to the singular locus of $\tilde{Y}$ :

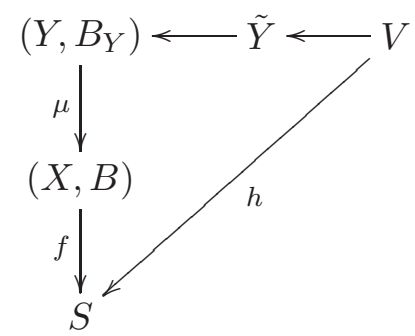

Let $\kappa_{V_{s}}$ be the induced Kodaira-Spencer class of $V \rightarrow S$, and let

$$
\begin{aligned}
\sigma_{V_{s}}: T_{S, s} & \rightarrow \operatorname{Hom}\left(H^{d, 0}\left(V_{s}\right), H^{d-1,1}\left(V_{s}\right)\right), \\
\sigma_{s}^{1}: T_{S, s} & \rightarrow \operatorname{Hom}\left(H^{0}\left(Y_{s},\left\lceil-B_{Y_{s}}\right\rceil\right), \operatorname{Ext}_{Y_{s}}^{1}\left(\Omega_{Y_{s}}^{1}\left\langle E_{s}\right\rangle, \mathcal{O}_{Y_{s}}\left(\left\lceil-B_{Y_{s}}\right\rceil\right)\right)\right)
\end{aligned}
$$




\section{THE MODULI B-DIVISOR}

be the maps induced by cup product with $\kappa_{V_{s}}$ and $\kappa_{s}$, respectively, where $d=\operatorname{dim}(X / S)$. The projective contraction $h$ is smooth over an open subset $S^{0}$ of $S$. Let $\Phi$ be the period map associated to the variation of polarized Hodge structure $\left(R^{d} h_{*} \mathbb{C}_{V}\right)_{\text {prim }} \otimes \mathcal{O}_{S^{0}}$.

Then the maps $\kappa_{s}, \sigma_{s}^{1}, \kappa_{V_{s}}, \sigma_{V_{s}}$ have the same kernel for general $s \in S$, equal to $T_{\Phi^{-1}(\Phi(s)), s} \subset T_{S, s}$.

Proof. (1) Since $\left(X_{s}, B_{X_{s}}\right)$ is a log variety with Kawamata log terminal singularities for general $s$, we have an isomorphism

$$
\mathcal{O}_{X_{s}} \stackrel{\sim}{\longrightarrow} \mu_{s_{*}} \mathcal{O}_{Y_{s}}\left(\left\lceil-B_{Y_{s}}\right\rceil\right) .
$$

In particular, the inclusion $\mathcal{O}_{Y_{s}} \rightarrow \mathcal{O}_{Y_{s}}\left(\left\lceil-B_{Y_{s}}\right\rceil\right)$ induces an isomorphism of global sections and identifies $\sigma_{s}^{1}$ with the composition

$$
T_{S, s} \rightarrow \operatorname{Ext}_{Y_{s}}^{1}\left(\Omega_{Y_{s}}^{1}\left\langle E_{s}\right\rangle, \mathcal{O}_{Y_{s}}\right) \stackrel{\lambda}{\rightarrow} \operatorname{Ext}_{Y_{s}}^{1}\left(\Omega_{Y_{s}}^{1}\left\langle E_{s}\right\rangle, \mathcal{O}_{Y_{s}}\left(\left\lceil-B_{Y_{s}}\right\rceil\right)\right)
$$

(2) $\operatorname{Ker}(\lambda) \subset \operatorname{Ker}\left(\operatorname{Ext}_{Y_{s}}^{1}\left(\Omega_{Y_{s}}^{1}\left\langle E_{s}\right\rangle, \mathcal{O}_{Y_{s}}\right) \rightarrow \operatorname{Ext}_{X_{s}}^{1}\left(\mu_{s *} \Omega_{Y_{s}}^{1}\left\langle E_{s}\right\rangle, \mathcal{O}_{X_{s}}\right)\right)$. Indeed, $\mathcal{O}_{Y_{s}}\left(\left\lceil-B_{Y_{s}}\right\rceil\right)$ is $\mu_{s *}$-acyclic by Kawamata-Viehweg vanishing. Since $X_{s}$ has rational singularities, $\mathcal{O}_{Y_{s}}$ is $\mu_{s_{*}}$-acyclic as well. The natural homomorphism $\mu_{s}^{*} \mu_{s *} \Omega_{Y_{s}}^{1}\left\langle E_{s}\right\rangle \rightarrow \Omega_{Y_{s}}^{1}\left\langle E_{s}\right\rangle$ induces the following commutative diagram.

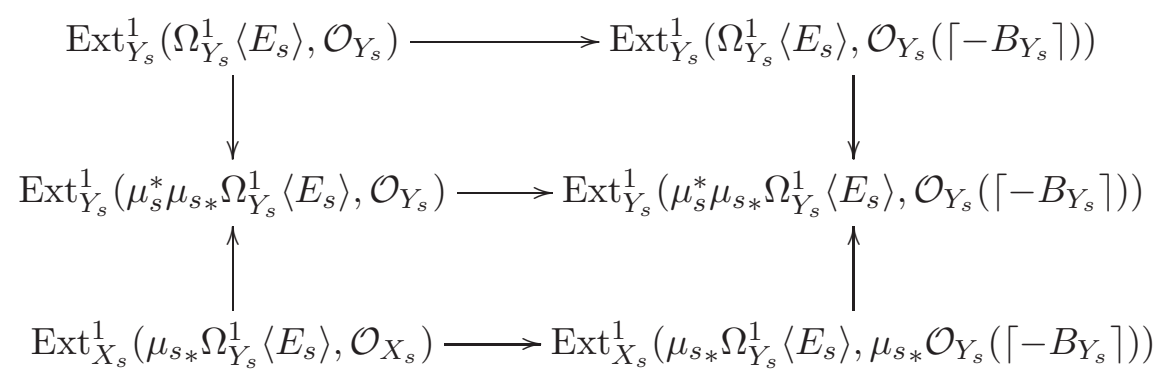

The lower vertical arrows are isomorphisms by Lemma 1.2, and the bottom horizontal arrow is an isomorphism by $\mathcal{O}_{X_{s}}=\mu_{s *} \mathcal{O}_{Y_{s}}\left(\left\lceil-B_{Y_{s}}\right\rceil\right)$. This implies the claim.

(3) $\operatorname{Ker}\left(\kappa_{s}\right)=\operatorname{Ker}\left(\sigma_{s}^{1}\right) \subseteq \operatorname{Ker}\left(\kappa_{X_{s}}\right)$ (the inclusion is an equality if $E$ is exceptional). Indeed, the inclusion $\operatorname{Ker}\left(\kappa_{s}\right) \subseteq \operatorname{Ker}\left(\sigma_{s}^{1}\right)$ is clear. Conversely, consider a tangent vector $t \in \operatorname{Ker}\left(\sigma_{s}^{1}\right)$, inducing a first-order infinitesimal deformation of $\left(Y_{s}, E_{s}\right)$ :

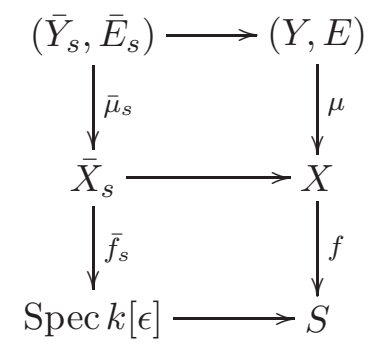

The class $\kappa_{s}(t)$ is represented by the short exact sequence

$$
0 \rightarrow \mathcal{O}_{Y_{s}} \rightarrow \Omega_{\bar{Y}_{s}}^{1}\left\langle\bar{E}_{s}\right\rangle \otimes \mathcal{O}_{Y_{s}} \rightarrow \Omega_{Y_{s}}^{1}\left\langle E_{s}\right\rangle \rightarrow 0
$$

By (2), the short exact sequence

$$
0 \rightarrow \mathcal{O}_{X_{s}} \rightarrow \mu_{s *}\left(\Omega_{\bar{Y}_{s}}^{1}\left\langle\bar{E}_{s}\right\rangle \otimes \mathcal{O}_{Y_{s}}\right) \rightarrow \mu_{s *} \Omega_{Y_{s}}^{1}\left\langle E_{s}\right\rangle \rightarrow 0
$$




\section{F. Ambro}

admits a splitting $u \in \operatorname{Hom}_{X_{s}}\left(\mu_{s_{*}}\left(\Omega_{\bar{Y}_{s}}^{1}\left\langle\bar{E}_{s}\right\rangle \otimes \mathcal{O}_{Y_{s}}\right), \mathcal{O}_{X_{s}}\right)$. It is enough to lift $u$ to $Y_{s}$. We may assume (cf. [Kaw85, Lemma 6.1]) that the horizontal arrows are injective and the vertical arrow is surjective in the diagram below.

$$
\begin{gathered}
\mathcal{H o m}_{\mathcal{O}_{X_{s}}}\left(\mu_{s *}\left(\Omega_{\bar{Y}_{s}}^{1}\left\langle\bar{E}_{s}\right\rangle \otimes \mathcal{O}_{Y_{s}}\right), \mathcal{O}_{X_{s}}\right) \longrightarrow \mathcal{H o m}_{\mathcal{O}_{X_{s}}}\left(\mu_{*} \Omega_{Y}^{1}\langle E\rangle \otimes \mathcal{O}_{X_{s}}, \mathcal{O}_{X_{s}}\right) \\
\uparrow \\
\mathcal{H o m}_{\mathcal{O}_{X}}\left(\Omega_{X}^{1}, \mathcal{O}_{X}\right) \longleftarrow \mathcal{H o m}_{\mathcal{O}_{X}}\left(\mu_{*} \Omega_{Y}^{1}\langle E\rangle, \mathcal{O}_{X}\right)
\end{gathered}
$$

Fix a point $x \in X_{s}$. Then $u$ lifts to a local section $\bar{u}$ of $\left(\mu_{*} \Omega_{Y}^{1}\langle E\rangle\right)^{\vee}$ near $x$. Since $\mu_{*} T_{Y}\langle-E\rangle$ is reflexive, we have $\mu_{*} T_{Y}\langle-E\rangle=\left(\mu_{*} \Omega_{Y}^{1}\langle E\rangle\right)^{\vee}$. Therefore $\bar{u}$ lifts to a section of $T_{Y}\langle-E\rangle$ in a neighborhood of $\mu^{-1}(x)$. In particular, $u$ lifts to a local splitting of $\kappa_{s}(t)$ in a neighborhood of $\mu^{-1}(x)$. Local liftings of $u$ are unique, hence they glue to a global splitting of $\kappa_{s}(t)$. Therefore $t \in \operatorname{Ker}\left(\kappa_{s}\right)$.

The inclusion $\operatorname{Ker}\left(\kappa_{s}\right) \subseteq \operatorname{Ker}\left(\kappa_{X_{s}}\right)$ follows from the following commutative diagram.

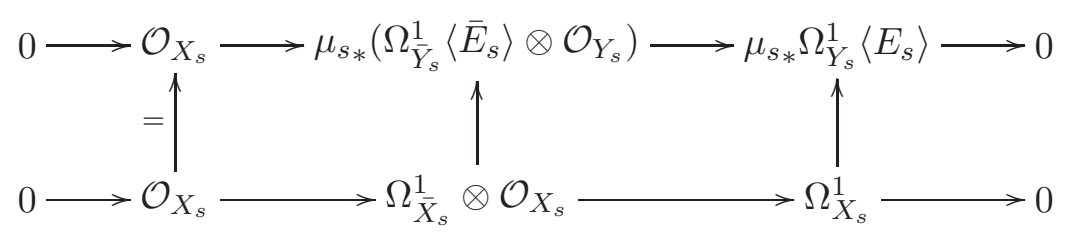

(4) The natural inclusion $\operatorname{Ker}\left(\kappa_{s}\right) \subseteq \operatorname{Ker}\left(\bigoplus_{i=0}^{b-1} \sigma_{s}^{i}\right) \subseteq \operatorname{Ker}\left(\sigma_{s}^{1}\right)$ and Proposition 1.3, part (iii) imply that the maps $\kappa_{s}, \kappa_{\tilde{Y}_{s}}, \kappa_{V_{s}}, \sigma_{s}^{1}, \sigma_{\tilde{Y}_{s}}, \sigma_{V_{s}}$ have the same kernel, for general $s \in S$. The inclusions

$$
\operatorname{Ker}\left(\kappa_{V_{s}}\right) \subseteq \operatorname{Ker}\left(d \Phi_{*}\right) \subseteq \operatorname{Ker}\left(\sigma_{V_{s}}\right)
$$

imply that the common kernel is the tangent space at $s$ of the fiber $\Phi^{-1}(\Phi(s))$ of $\Phi$.

Theorem 2.2. There exist dominant morphisms $\tau: \bar{S} \rightarrow S$ and $\varrho: \bar{S} \rightarrow S^{!}$, with $\tau$ generically finite and $\bar{S}, S^{!}$nonsingular, and there exist a $\log$ variety $\left(X^{!}, B^{!}\right)$with $K_{X^{!}}+B^{!} \sim_{\mathbb{Q}} 0$ and a projective contraction $f^{!}: X^{!} \rightarrow S^{!}$

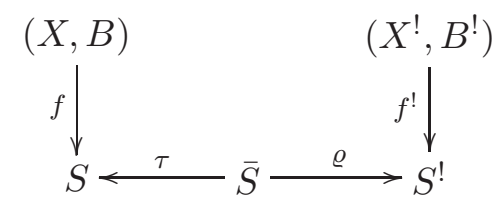

satisfying the following properties:

(i) there exists an open dense subset $U \subset \bar{S}$ and an isomorphism

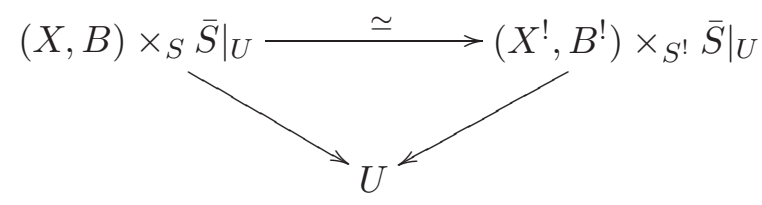

(ii) $\kappa_{s}$ ! is injective for general points $s^{!} \in S^{!}$.

Proof. We may assume that $h: V \rightarrow S$ is a smooth projective morphism. Let $S \subset \bar{S}$ be a nonsingular compactification with simple normal crossing boundary. By [Gri70], the period map $\Phi$ of the variation of polarized Hodge structure $\left(R^{d} h_{*} \mathbb{C}_{V}\right)_{\text {prim }} \otimes \mathcal{O}_{S}$ extends to a proper analytic morphism defined on an open subvariety of $\bar{S}$. By [Kaw83], $\Phi$ is bimeromorphic to a rational map

$$
\Phi: S \rightarrow S^{!}
$$




\section{THE MODULI B-DIVISOR}

and we may further shrink $S$ so that $\Phi$ is regular everywhere on $S$. After we replace $S$ by an étale open set, the following properties hold:

(a) $(Y, E) \rightarrow S$ is a smooth relative pair and $\mu_{s *} \mathcal{O}_{Y_{s}}=\mathcal{O}_{X_{s}}$ for every $s \in S$;

(b) $\operatorname{Ext}_{Y_{s}}^{1}\left(\Omega_{Y_{s}}^{1}\left\langle E_{s}\right\rangle, \mathcal{O}_{Y_{s}}\right)$ and $\operatorname{Ext}_{X_{s}}^{1}\left(\Omega_{X_{s}}^{1}, \mathcal{O}_{X_{s}}\right)$ have constant dimension for $s \in S$;

(c) $\operatorname{Ker}\left(\kappa_{s}\right)=T_{\Phi^{-1}(\Phi(s))}$ for every $s \in S$;

(d) $\Phi: S \rightarrow S^{!}$has a section $i: S^{!} \rightarrow S$.

By base change via the section $i$, we induce a family on $S^{!}$:

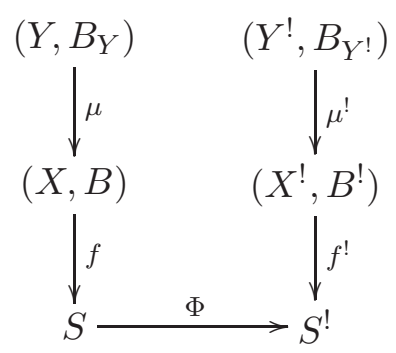

According to Proposition 2.1 and its proof, the families $(Y, E) \rightarrow S$ and $X \rightarrow S$ are first-order infinitesimally trivial when restricted to the fibers of $\Phi$. The same holds for the map $Y \rightarrow X$ over $S$, by (a) (see [Ran89]). By [Gra74] and [Kaw78], the family

$$
(Y, E) \rightarrow X \rightarrow S
$$

is locally trivial when restricted to the fibers of $\Phi$. Consider the subfunctor of

$$
\operatorname{Isom}_{S}\left(X, X^{!} \times_{S^{!}} S\right) \times \operatorname{Isom}_{S}\left((Y, E),\left(Y^{!}, E^{!}\right) \times_{S^{!}} S\right),
$$

making the obvious diagrams commutative. This subfunctor is representable by a scheme $I / S$, and the map $I \rightarrow S$ is surjective from the above considerations. After replacing $S$ (and $S^{!}$accordingly) by an étale open subset, we may assume that $I / S$ has a section. Consequently, we obtain global isomorphisms as below.

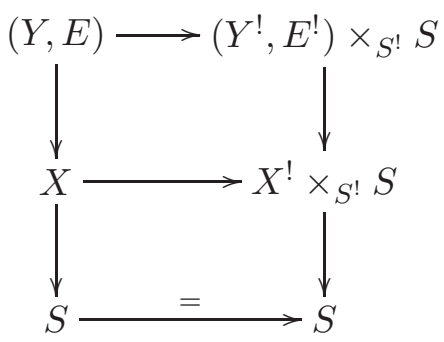

Since $(X, B)$ has Kawamata log-terminal singularities and $B$ is effective, we have $B=\mu_{*} E$ and $B^{!} \times{ }_{S^{!}} S=\left(\mu^{!} \times{ }_{S^{!}} 1_{S}\right)_{*}\left(E^{!} \times{ }_{S^{!}} S\right)$. Therefore $X \rightarrow X^{!} \times{ }_{S^{!}} S$ is in fact an isomorphism of pairs over $S$

$$
(X, B) \stackrel{\sim}{\longrightarrow}\left(X^{!}, B^{!}\right) \times_{S^{!}} S .
$$

By (c), the Kodaira-Spencer class $\kappa_{s}$ ! is injective for $s^{!} \in S^{!}$.

\section{Lc-trivial fibrations}

Recall [Amb02] that an lc-trivial fibration $f:(X, B) \rightarrow Y$ consists of a contraction $f: X \rightarrow Y$ of proper normal varieties and a log pair $(X, B)$, subject to the following conditions:

(1) $(X, B)$ has Kawamata log terminal singularities over the generic point of $Y$;

(2) $\operatorname{rank} f_{*} \mathcal{O}_{X}(\lceil\mathbf{A}(X, B)\rceil)=1$, where $\mathbf{A}(X, B)$ is the discrepancy $\mathbb{R}$-b-divisor of $(X, B)$; 


\section{F. AmBro}

(3) there exist a positive integer $r$, a rational function $\varphi \in k(X)^{\times}$and a $\mathbb{Q}$-Cartier divisor $D$ on $Y$ such that

$$
K+B+\frac{1}{r}(\varphi)=f^{*} D
$$

The lc-trivial fibration $f:(X, B) \rightarrow Y$ induces $\mathbb{Q}$-b-divisors B and $\mathbf{M}$ of $Y$, called the discriminant and moduli $\mathbb{Q}$-b-divisor respectively. By [Amb02, Theorem 0.2], the moduli $\mathbb{Q}$-b-divisor is b-nef. This means that there exists a proper birational model $Y^{\prime}$ of $Y$ such that $\mathbf{M}=\overline{\mathbf{M}_{Y^{\prime}}}$ and $\mathbf{M}_{Y^{\prime}}$ is a nef $\mathbb{Q}$-Cartier divisor.

Proposition 3.1. Let $f:(X, B) \rightarrow Y$ be an lc-trivial fibration. Let $\varrho: Y^{\prime} \rightarrow Y$ be a surjective morphism from a proper normal variety $Y^{\prime}$, and let $f^{\prime}:\left(X^{\prime}, B_{X^{\prime}}\right) \rightarrow Y^{\prime}$ be an lc-trivial fibration induced by base change.

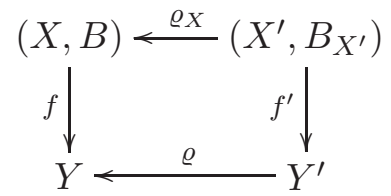

Let $\mathbf{M}$ and $\mathbf{M}^{\prime}$ be the corresponding moduli $\mathbb{Q}$-b-divisors. Then

$$
\varrho^{*} \mathbf{M}=\mathbf{M}^{\prime} .
$$

Proof. (1) To define the induced lc-trivial fibration, we may assume that $X^{\prime}$ is the normalization of the main component of $X \times_{Y} Y^{\prime}$. Also, we may assume that $X$ is nonsingular. Then there exists a canonical divisor $K_{X^{\prime}}$ on $X^{\prime}$ such that $A=K_{X^{\prime}}-\varrho_{X}^{*} K$ is an $f^{\prime}$-vertical Weil divisor. Define $B_{X^{\prime}}=\varrho_{X}^{*} B-A$, so that

$$
K_{X^{\prime}}+B_{X^{\prime}}+\frac{1}{r}\left(\varrho_{X}^{*} \varphi\right)=f^{\prime *}\left(\varrho^{*} D\right) .
$$

The moduli $\mathbb{Q}$-b-divisor $\mathbf{M}^{\prime}$ of the lc-trivial fibration $\left(X^{\prime}, B_{X^{\prime}}\right)$ is independent of the above choice of $K_{X^{\prime}}$, by [Amb02, Remark 3.3].

(2) We have $\varrho^{*} \mathbf{M}=\mathbf{M}^{\prime}$ if $\varrho$ is generically finite. This is clear if $\varrho$ is birational. If $\varrho$ is a finite morphism, $\varrho^{*} \mathbf{M}=\mathbf{M}^{\prime}$ by [Amb99, Theorem 3.2] and [Amb02, Remark 3.3].

(3) The general case follows from (1) and the compatibility with base change of the canonical extension of a variation of Hodge structure with unipotent local monodromies at infinity (same argument as in the proof of [Amb02, Theorem 2.7]).

Definition 3.2. A $\mathbb{Q}$-b-divisor $\mathbf{M}$ of $Y$ is called $b$-nef and good if there exists a proper birational model $Y^{\prime}$ of $Y$, endowed with a proper contraction $h: Y^{\prime} \rightarrow Z$, such that:

(1) $\mathbf{M}_{Y^{\prime}} \sim_{\mathbb{Q}} h^{*} H$, for some nef and big $\mathbb{Q}$-divisor $H$ of $Z$;

(2) $\mathbf{M}=\overline{\mathbf{M}_{Y^{\prime}}}$.

Theorem 3.3. Let $f:(X, B) \rightarrow Y$ be an lc-trivial fibration such that the geometric generic fiber $X_{\bar{\eta}}=X \times_{Y} \operatorname{Spec}(\overline{k(Y)})$ is a projective variety and $B_{\bar{\eta}}$ is effective. Then there exists a diagram

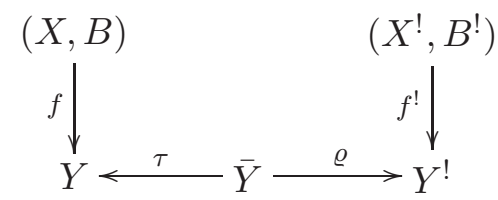

satisfying the following properties:

(i) $f^{!}:\left(X^{!}, B^{!}\right) \rightarrow Y^{!}$is an lc-trivial fibration;

(ii) $\tau$ is generically finite and surjective and $\varrho$ is surjective; 


\section{THE MODULI B-DIVISOR}

(iii) there exists a nonempty open subset $U \subset \bar{Y}$ and an isomorphism

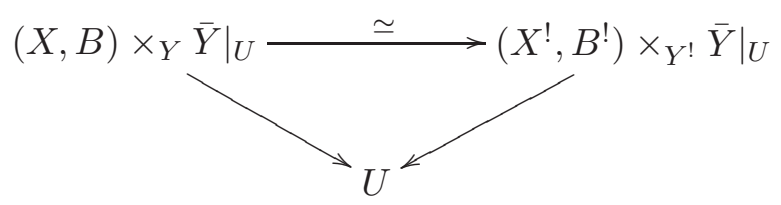

(iv) let $\mathbf{M}, \mathbf{M}^{!}$be the corresponding moduli $\mathbb{Q}$-b-divisors. Then $\mathbf{M}^{!}$is b-nef and big and $\tau^{*} \mathbf{M}=$ $\varrho^{*}\left(\mathbf{M}^{!}\right)$.

In particular, the moduli $\mathbb{Q}$-b-divisor $\mathbf{M}$ is b-nef and good.

Proof. Note that the restriction of $f:(X, B) \rightarrow Y$ to an appropriate open subset of $Y$ satisfies the assumptions of $\S 2$.

(1) Assume that $\kappa_{s}$ is injective for sufficiently general points $s \in Y$. Then $\mathbf{M}$ is b-nef and big.

Indeed, let $\mu: X^{\prime} \rightarrow X$ be an equivariant resolution of $X$ with respect to $\operatorname{Sing}(X) \cup \operatorname{Supp}(B)$. Let $\tilde{X}^{\prime} \rightarrow X^{\prime}$ be the normalization of $X^{\prime}$ in $k\left(X^{\prime}\right)(\sqrt[b]{\varphi})$, let $V \rightarrow \tilde{X}^{\prime}$ be an equivariant resolution with respect to the singular locus of $\tilde{X}^{\prime}$, and let $f^{\prime}: X^{\prime} \rightarrow Y$ be the induced morphism:

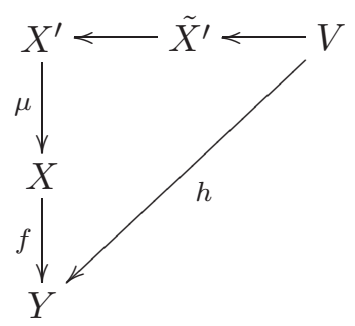

After a generically finite base change of $Y$, we may assume that $\mathbf{M}$ descends to $Y$ and $V / Y$ is birational to $V^{\prime} / Y$ which has simple normal crossings degeneration and is semistable in codimension 1. Under these assumptions, we infer by [Amb02, Lemma 5.2] that $\mathbf{M}_{Y}$ is a nef Cartier divisor and $\mathcal{O}_{Y}\left(\mathbf{M}_{Y}\right)$ is isomorphic to the Schmid extension

$$
\overline{\mathcal{F}}^{(1)}=f_{*}^{\prime} \mathcal{O}_{X^{\prime}}\left(\left\lceil-B_{X^{\prime}}+f^{\prime *} B_{Y}+f^{\prime *} \mathbf{M}_{Y}\right\rceil\right) .
$$

By Proposition 2.1, $\sigma_{s}^{1}$ and $\kappa_{s}$ have the same kernel, hence $\sigma_{s}^{1}$ is injective. By Proposition 1.3, the invertible sheaf $\overline{\mathcal{F}}^{(1)}$ has positive self-intersection. Therefore $\mathbf{M}_{Y}$ is a nef and big Cartier divisor, hence $\mathbf{M}=\overline{\mathbf{M}_{Y}}$ is b-nef and big.

(2) We may assume that the base space $Y$ is nonsingular. By Theorem 2.2, there exists a diagram

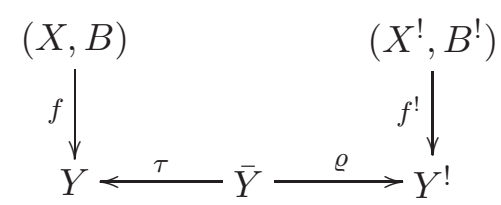

satisfying the following properties:

(a) $\tau$ is generically finite and surjective and $\varrho$ is surjective;

(b) $f^{!}:\left(X^{!}, B^{!}\right) \rightarrow Y^{!}$is an lc-trivial fibration;

(c) there exists a nonempty open subset $U \subset \bar{Y}$ and an isomorphism

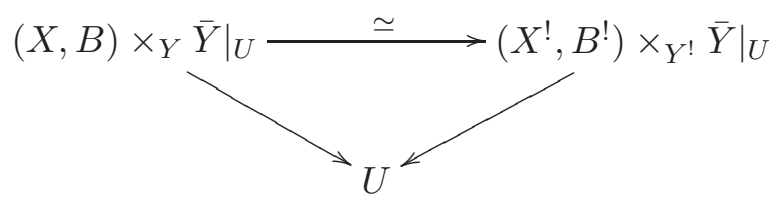




\section{F. AmBro}

The moduli $\mathbb{Q}$-b-divisor $\mathbf{M}^{!}$is b-nef and big, by (1). Let $\bar{f}: \bar{X} \rightarrow \bar{Y}$ be a contraction which is birationally induced by both $f$ and $f^{!}$, and let $\left(\bar{X} / \bar{Y}, B_{\bar{X}}\right)$ and $\left(\bar{X} / \bar{Y}, B_{\bar{X}}^{!}\right)$be the lc-trivial fibrations induced by base change. From (c), there exists a $\mathbb{Q}$-Cartier divisor $L$ on $\bar{Y}$ such that $B_{\bar{X}}=B_{\bar{X}}+\bar{f}^{*} L$. Therefore the lc-trivial fibrations $\left(\bar{X} / \bar{Y}, B_{\bar{X}}\right)$ and $\left(\bar{X} / \bar{Y}, B_{\bar{X}}^{!}\right)$have the same moduli $\mathbb{Q}$-b-divisor (see [Amb02, Remark 3.3]). We conclude by Proposition 3.1 that $\tau^{*}(\mathbf{M})=\varrho^{*}\left(\mathbf{M}^{!}\right)$.

Proposition 3.4. Let $f: X \rightarrow Y$ be a morphism of projective manifolds, and let $\Sigma_{Y} \subset Y$ be a simple normal crossings divisor such that:

(i) $f$ is smooth over $Y \backslash \Sigma_{Y}$;

(ii) $f$ is semistable over codimension-1 points of $Y$.

Let $\mathcal{L} \subset f_{*} \omega_{X / Y}$ be a direct summand invertible sheaf such that $\mathcal{L} \equiv 0$. Then $\mathcal{L}^{\otimes r} \simeq \mathcal{O}_{X}$ for some positive integer $r$.

Proof. Consider the variation of polarized Hodge structure of weight $d$

$$
H=R^{d} f_{*} \mathbb{C}_{X^{0}} \otimes \mathcal{O}_{Y^{0}},
$$

where $Y^{0}=Y \backslash \Sigma_{Y}$ and $d=\operatorname{dim}(X / Y)$. The sheaf

$$
\left.\left.\mathcal{L}\right|_{Y^{0}} \subset f_{*} \omega_{X / Y}\right|_{Y^{0}}=F^{d} H
$$

has an induced Hermitian metric $h$ with semipositive curvature form $\Theta$. We claim that the curvature is trivial. Indeed, let $v \in T_{Y, s}$ be a tangent vector at a point $s \in Y^{0}$. There exists a projective curve $C \subset Y$ such that $T_{C, s}=\mathbb{C} v$, and let $\nu: C^{\nu} \rightarrow C$ be its normalization. Since the local monodromies of $H$ at infinity are unipotent by assumption, we infer by [Kaw81] that

$$
\operatorname{deg}\left(\left.\mathcal{L}\right|_{C}\right)=\frac{\sqrt{-1}}{2 \pi} \int_{\nu^{-1}\left(Y^{0}\right)} \nu^{*} \Theta .
$$

Since $\mathcal{L}$ is numerically trivial, we have $\nu^{*} \Theta=0$. Since $\nu$ is an isomorphism near $s$, we conclude that $v$ lies is a null direction of $\Theta$.

Therefore $\Theta$ is trivial, i.e. $\left.\mathcal{L}\right|_{Y^{0}} \subset H$ is a local subsystem. By Deligne, there exists a positive integer $r$ such that $\left(\left.\mathcal{L}\right|_{Y^{0}}\right)^{\otimes r}$ is a trivial local system. By [Kaw81], $f_{*} \omega_{X / Y}$ is the canonical extension of $\left.f_{*} \omega_{X / Y}\right|_{Y^{0}}$. The same property holds for its direct summand $\mathcal{L}$. Since the local monodromies are unipotent, the canonical extension commutes with tensor products. Therefore $\mathcal{L}^{\otimes r} \simeq \mathcal{O}_{X}$.

Theorem 3.5. Let $f:(X, B) \rightarrow Y$ be an lc-trivial fibration. If $\mathbf{M}$ is b-numerically trivial, then $\mathbf{M} \sim \mathbb{Q} 0$.

Proof. This is similar to the proof of [Amb02, Theorem 0.1]. After a finite base change [Amb02, Lemma 5.1], we may assume that the induced root fiber space $h: V \rightarrow Y$ is semistable in codimension 1 and $\mathbf{M}$ descends to $Y$. By construction, the invertible sheaf $\mathcal{O}_{Y}\left(\mathbf{M}_{Y}\right) \subset h_{*} \omega_{V / Y}$ is a direct summand, and $\mathbf{M}_{Y} \equiv 0$.

We conclude by Proposition 3.4 that $\mathbf{M}_{Y}$ is torsion. Therefore $\mathbf{M}$ is torsion.

\section{Applications}

Theorem 4.1. Let $(X, B)$ be a projective log variety with Kawamata log terminal singularities, let $f: X \rightarrow Y$ be a contraction to a proper normal variety $Y$, and let $\omega$ be a $\mathbb{Q}$-Cartier divisor on $Y$ such that

$$
K+B \sim_{\mathbb{Q}} f^{*} \omega .
$$

Then there exists a $\mathbb{Q}$-Weil divisor $B_{Y}$ such that $\left(Y, B_{Y}\right)$ is a log variety with Kawamata log terminal singularities and $\omega \sim_{\mathbb{Q}} K_{Y}+B_{Y}$. 


\section{THE MODULI B-DIVISOR}

Proof. We may write $K+B+(1 / r)(\varphi)=f^{*} \omega$, where $r$ is a positive integer and $\varphi$ is a rational function on $X$. Thus, $f:(X, B) \rightarrow Y$ is an lc-trivial fibration. Denote by $\mathbf{B}$ and $\mathbf{M}$ the induced discriminant and moduli $\mathbb{Q}$-b-divisors. The assumptions of Theorem 3.3 are satisfied, so we may find a high resolution $\sigma: Y^{\prime} \rightarrow Y$ such that $\mathbf{M}=\overline{\mathbf{M}_{Y^{\prime}}}$ and

$$
\mathbf{M}_{Y^{\prime}} \sim \mathbb{Q} h^{*} A,
$$

where $h: Y^{\prime} \rightarrow Z$ is a contraction to a normal projective variety $Z$ and $A$ is a nef and big divisor on $Z$. Consider the lc-trivial fibration induced by base change with $\sigma$ :

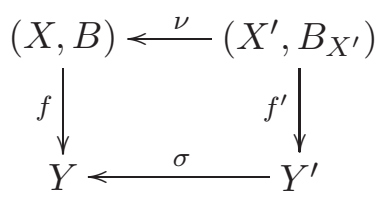

Then $\sigma^{*} \omega=K_{Y^{\prime}}+\mathbf{B}_{Y^{\prime}}+\mathbf{M}_{Y^{\prime}}$. Since $\mathbf{M}$ descends to $Y^{\prime}$, inversion of adjunction holds for $f^{\prime}$ (see [Amb02, Theorem 3.1]). Therefore $\left(Y^{\prime}, \mathbf{B}_{Y^{\prime}}\right)$ is a log pair with Kawamata log terminal singularities. We may find an effective $\mathbb{Q}$-divisor $E$ on $Y^{\prime}$ such that $\mathbf{M}_{Y^{\prime}} \sim_{\mathbb{Q}} E$ and $\left(Y^{\prime}, \mathbf{B}_{Y^{\prime}}+E\right)$ has Kawamata $\log$ terminal singularities. If we set $B_{Y}=\sigma_{*}\left(\mathbf{B}_{Y^{\prime}}+E\right)$, then

$$
\sigma^{*}\left(K_{Y}+B_{Y}\right)=K_{Y^{\prime}}+\mathbf{B}_{Y^{\prime}}+E \sim \sigma^{*} \omega,
$$

and $\left(Y, B_{Y}\right)$ is a log pair with Kawamata log terminal singularities. Since $B$ is effective, $\mathbf{B}_{Y}=\sigma_{*} \mathbf{B}_{Y^{\prime}}$ is effective. Therefore $B_{Y}$ is effective, i.e. $\left(Y, B_{Y}\right)$ is a $\log$ variety.

Theorem 4.2. Let $(X, B)$ be a projective $\log$ variety with Kawamata log terminal singularities such that $K+B \equiv 0$. Then $K+B \sim_{\mathbb{Q}} 0$.

Proof. The variety $X$ has rational singularities since $(X, B)$ has Kawamata log terminal singularities. Therefore the Albanese map of $X$ is a morphism [Kaw85].

We use induction on $\operatorname{dim}(X)$. If $q(X)=0$, the numerically trivial divisor $K+B$ is certainly a torsion divisor. Assume now that $q(X)>0$. Let $f: X \rightarrow Y$ be the Stein factorization of the Albanese map of $X$. The geometric generic fiber $\left(X_{\bar{\eta}}, B_{\bar{\eta}}\right)$ satisfies the same properties as $(X, B)$ and $\operatorname{dim} X_{\bar{\eta}}<\operatorname{dim} X$. Therefore $K_{X_{\bar{\eta}}}+B_{\bar{\eta}} \sim_{\mathbb{Q}} 0$, by induction. In particular, $K+B$ is numerically trivial and $\mathbb{Q}$-linearly equivalent to an $f$-vertical divisor.

Therefore we may choose a sufficiently high resolution $\mu: Y^{\prime} \rightarrow Y$ and a diagram induced by base change

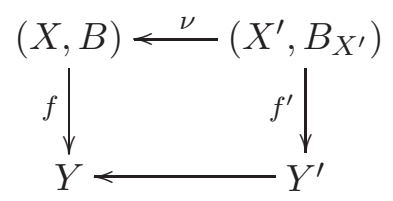

such that $f^{\prime}:\left(X^{\prime}, B_{X^{\prime}}\right) \rightarrow Y^{\prime}$ is an lc-trivial fibration and every prime divisor on $X^{\prime}$ is exceptional on $X$ if it is exceptional on $Y^{\prime}$. We have

$$
\nu^{*}(K+B)+f^{\prime *}\left(\mathbf{B}_{Y^{\prime}}^{-}\right)=f^{\prime *}\left(K_{Y^{\prime}}+\mathbf{B}_{Y^{\prime}}^{+}+\mathbf{M}_{Y^{\prime}}\right),
$$

where $\mathbf{B}_{Y^{\prime}}=\mathbf{B}_{Y^{\prime}}^{+}-\mathbf{B}_{Y^{\prime}}^{-}$is the decomposition of the discriminant on $Y^{\prime}$ into positive and negative components. The effective $\mathbb{Q}$-divisor $f^{\prime *}\left(\mathbf{B}_{Y^{\prime}}^{-}\right)$is exceptional on $X$, since it is supported by the negative part of $B_{X^{\prime}}$ and by $f^{\prime}$-exceptional divisors.

By [Uen75, Theorem 10.3], $\kappa\left(Y^{\prime}, K_{Y^{\prime}}\right) \geqslant 0$. Since $\mathbf{M}$ is b-nef and good, we also have $\kappa\left(Y^{\prime}, \mathbf{M}_{Y^{\prime}}\right) \geqslant 0$. Therefore $\kappa(X, K+B) \geqslant 0$, hence $K+B \sim_{\mathbb{Q}} 0$.

Theorem 4.3. Let $(X, B)$ be a projective $\log$ variety with Kawamata log terminal singularities, such that the $\log$ canonical class $K+B$ is nef, of nef dimension $n$. Assume that the Log Minimal Model Program and the Log Abundance Conjecture are valid in dimension $n$. 


\section{F. AmBro}

Then the linear system $|k(K+B)|$ is base point free for sufficiently large and divisible positive integers $k$.

Proof. The argument of [Amb03, Theorem 5.1] is still valid, provided we replace the references [Kaw85, Theorem 8.2] and [Amb03, Theorem 4.5] with [Kaw85, Theorem 4.2] and [Amb03, Theorem 3.3], respectively.

For the rest of this section, we generalize some results of Viehweg [Vie83a] and Kawamata [Kaw85] on Ueno's K Conjecture [Uen75].

Proposition 4.4. Let $f:(X, B) \rightarrow Y$ be an lc-trivial fibration of normal projective varieties such that there exists an isomorphism

$$
\Phi:\left.(X, B)\right|_{U} \stackrel{\sim}{\longrightarrow}\left(F, B_{F}\right) \times U \text { over } U,
$$

where $U \subset X$ is a nonempty open subset. Let

$$
Y^{0}=Y \backslash\left\{\operatorname{Sing}(Y) \cup \operatorname{Supp}\left(\mathbf{B}_{Y}\right) \cup f\left(\operatorname{Supp}\left(B_{-}^{v}\right)\right)\right\},
$$

where $B_{-}^{v}$ is the negative and vertical part of $B$. Then $\Phi$ extends to an isomorphism $\Phi:\left.(X, B)\right|_{Y^{0}} \stackrel{\sim}{\longrightarrow}$ $\left(F, B_{F}\right) \times Y^{0}$ over $Y^{0}$.

Proof. (1) Let $E$ be a prime divisor on $X$ such that $\operatorname{codim}_{X}(f(E)) \geqslant 2$. Then $f(E) \cap Y^{0}=\emptyset$.

Indeed, the assumption implies $\mathbf{M} \sim \mathbb{Q} 0$. In particular, $\mathbf{M}$ descends to $Y$, i.e. inversion of adjunction [Amb02, Theorem 3.1] holds for $f$. If $B$ is negative at $E$, then $f(E) \cap Y^{0}=\emptyset$. Otherwise, the $\log$ discrepancy of $(X, B)$ at the generic point of $E$ is $1-\operatorname{mult}_{E}(B) \leqslant 1$. By inversion of adjunction, the minimal $\log$ discrepancy of $\left(Y, \mathbf{B}_{Y}\right)$ at the generic point of $f(E)$ is at most 1 . Since $B$ is effective, $\mathbf{B}_{Y}$ is effective. Since $f(E)$ is a subvariety of codimension at least 2 , it must be contained either in the singular locus of $Y$, or in the support of $\mathbf{B}_{Y}$.

(2) The rational map $\Phi:\left.X\right|_{Y^{0}} \rightarrow F \times Y^{0}$, and its inverse, are isomorphisms in codimension 1. Moreover, $\left.B\right|_{Y^{0}}$ is a horizontal $\mathbb{Q}$-divisor.

By assumption, $\Phi$ is an isomorphism above the generic point of $Y^{0}$. By (1), a prime divisor on $\left.X\right|_{Y^{0}}$ is vertical over $Y^{0}$ if and only if it maps onto a prime divisor of $Y^{0}$. Therefore we may assume that $Y$ is a curve, and it suffices to show that $\Phi$ induces a birational map $X_{P} \rightarrow F \times P$ for every point $P \in Y \backslash \operatorname{Supp}\left(\mathbf{B}_{Y}\right)$.

We have $\mathbf{A}\left(X, B+f^{*} P\right)=\mathbf{A}\left(F \times Y, B_{F} \times Y+F \times \mathbf{B}_{Y}+F \times P\right)$. The log variety

$$
\left(F \times Y, B_{F} \times Y+F \times P\right)=\left(F, B_{F}\right) \times(Y, P)
$$

has $\log$ canonical singularities near $F \times P$, with $F \times P$ the unique lc place over $P$. Since $\mathbf{B}_{Y}=0$ near $P,\left(X, B+f^{*} P\right)$ has $\log$ canonical singularities near $X_{P}$, with $F \times P$ the unique lc place over $P$. Since $B$ is effective at the components of $X_{P}$, this implies that $X_{P}$ is a reduced prime divisor and $\Phi$ induces a birational map $X_{P} \rightarrow F \times P$. Moreover, $B$ has multiplicity zero at $X_{P}$.

(3) We have an induced isomorphism $\Phi: V_{1} \stackrel{\sim}{\longrightarrow} V_{2}$, where $V_{1}, V_{2}$ are big open subsets of $\left.X\right|_{Y^{0}}$ and $F \times Y^{0}$, respectively. Fix a point on $Y^{0}$ with a local chart $\left(\Delta ; t_{1}, \ldots, t_{n}\right)$. The sections

$$
p^{*} \frac{\partial}{\partial t_{i}} \in H^{0}\left(F \times \Delta,\left(p^{*} \Omega_{Y}^{1}\right)^{\vee}\right)
$$

lift to vector fields

$$
\mathfrak{a}_{i} \in H^{0}\left(F \times \Delta, T_{F \times Y}\right) .
$$

The sheaves $T_{X}, T_{F \times Y},\left(p^{*} \Omega_{Y}^{1}\right)^{\vee}$ and $\left(f^{*} \Omega_{Y}^{1}\right)^{\vee}$ are reflexive, hence $\Phi$ induces vector fields

$$
\mathfrak{a}_{i}^{\prime} \in H^{0}\left(\left.X\right|_{\Delta}, T_{X}\right)
$$


which lift

$$
f^{*} \frac{\partial}{\partial t_{i}} \in H^{0}\left(\left.X\right|_{\Delta},\left(f^{*} \Omega_{Y}^{1}\right)^{\vee}\right) .
$$

By [GK64], these vector fields define one-parameter groups of automorphisms in a neighborhood of the fixed fiber, which in turn define a trivialization of $f$ near the fixed fiber. By construction, the oneparameter groups of $\mathfrak{a}_{i}$ and $\mathfrak{a}_{i}^{\prime}$ are compatible via $\Phi$. Therefore $\Phi$ is an isomorphism near the fixed fiber.

As for the boundary, note that $\left.B\right|_{Y^{0}}$ is horizontal over $Y^{0}$. Since $\Phi$ preserves the boundaries over the generic point of $Y^{0}$, the isomorphism $\Phi:\left.X\right|_{Y^{0}} \stackrel{\sim}{\longrightarrow} F \times Y^{0}$ satisfies $\Phi(B)=B_{F} \times Y^{0}$.

Example 4.5. Let $Y$ be a surface with DuVal singularities. Then the minimal resolution $f: X \rightarrow Y$ is an lc-trivial fibration with $\mathbf{B}_{Y}=0$ and $\mathbf{M}=0$. It is an isomorphism only outside the DuVal singularities.

Proposition 4.6. Let $(X, B)$ be a proper log variety with Kawamata log terminal singularities, such that $\kappa(X, K+B) \geqslant 0$. Let $\operatorname{Aut}^{0}(X, B)$ be the connected component of the identity of the group scheme

$$
\operatorname{Aut}(X, B)=\left\{\sigma \in \operatorname{Aut}(X) ; \sigma_{*}(B)=B\right\}
$$

Then $\operatorname{Aut}^{0}(X, B)$ is an Abelian variety.

Proof. It is known that $\operatorname{Aut}^{0}(X)$ is an algebraic group [Gro62, MO67], so its closed subgroup $\operatorname{Aut}^{0}(X, B)$ is an algebraic group. Assume by contradiction that $\operatorname{Aut}^{0}(X, B)$ contains a linear algebraic group. Then $\operatorname{Aut}^{0}(X, B)$ contains a connected one-dimensional linear group $G=\mathbb{G}_{m}$ or $\mathbb{G}_{a}$ (see $[\operatorname{Ros} 56])$.

The closed subset $\operatorname{Sing}(X) \cup \operatorname{Supp}(B)$ is $G$-invariant. By [Ros56, Theorem 10], there exists a $G$-invariant open subset $U \subset X \backslash(\operatorname{Sing}(X) \cup \operatorname{Supp}(B))$ and a $G$-invariant isomorphism

$$
U \stackrel{\sim}{\rightarrow} G \times V,
$$

where $G$ acts on $G \times V$ only on the first factor, by translations. In particular, $V$ is nonsingular. Choose a compactification $V \subset Y$ such that $Y \backslash V$ is a simple normal crossings divisor. By Hironaka's resolution of singularities, there exists a diagram

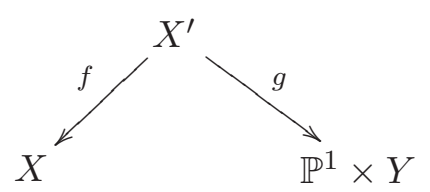

such that $f$ is an isomorphism over $U, g$ is an isomorphism over $G \times V, X^{\prime}$ is proper and nonsingular, and

$$
f^{-1}(X \backslash U)=g^{-1}\left(\mathbb{P}^{1} \times Y \backslash G \times V\right)=\sum E_{i}
$$

is a simple normal crossings divisor on $X^{\prime}$. Let $f^{*}(K+B)=K+B_{Y}$, and let $B_{Y}^{+}$be the positive part of $B_{Y}$. We have $\left\lfloor B_{Y}^{+}\right\rfloor=0$, since $(X, B)$ has Kawamata log terminal singularities. Since $B$ is effective, we have

$$
\kappa\left(X^{\prime}, K_{X^{\prime}}+B_{Y}^{+}\right)=\kappa(X, K+B) \geqslant 0 .
$$

In particular, $\kappa\left(\mathbb{P}^{1} \times Y, g_{*}\left(B_{Y}^{+}\right)\right) \geqslant 0$. But

$$
\left(\mathbb{P}^{1} \times Y, g_{*}\left(B_{Y}^{+}\right)\right)=\left(\mathbb{P}^{1}, B_{\mathbb{P}^{1}}\right) \times\left(Y, B_{Y}\right),
$$

where $B_{\mathbb{P} 1}$ and $B_{Y}$ are boundaries supported by $\mathbb{P}^{1} \backslash G$ and $Y \backslash V$, respectively. In particular, $\kappa\left(\mathbb{P}^{1}, K_{\mathbb{P}^{1}}+B_{\mathbb{P}^{1}}\right) \geqslant 0$. Since $B_{\mathbb{P}^{1}}$ is a boundary, this implies that $G=\mathbb{G}_{m}$ and $B_{\mathbb{P}^{1}}$ is the reduced sum of two points. This contradicts $\left\lfloor B_{Y}^{+}\right\rfloor=0$. 


\section{F. Ambro}

Theorem 4.7. Let $f:(X, B) \rightarrow Y$ be an lc-trivial fibration such that $X_{\bar{\eta}}$ is projective, $B$ is effective over a big open subset of $Y, \mathbf{B}_{Y}=0$, and $\mathbf{M} \sim_{\mathbb{Q}} 0$. Then there exists a finite Galois covering $\tau: Y^{\prime} \rightarrow Y$ such that:

(i) $\tau$ is étale in codimension 1;

(ii) there exists a nonempty open subset $U \subset Y^{\prime}$ and an isomorphism $(X, B) \times\left._{Y} Y^{\prime}\right|_{U} \stackrel{\sim}{\longrightarrow}\left(F, B_{F}\right) \times$ $\left.Y^{\prime}\right|_{U}$ over $U$.

Proof. (1) The fibers of $f$ are reduced over a big open subset of $Y$. Indeed, we may assume that $Y$ is a curve. Let $P \in Y$ be a point and let $f^{*} P=\sum_{i} m_{i} E_{i}$. We have

$$
1=1-\operatorname{mult}_{P}\left(\mathbf{B}_{Y}\right) \leqslant \min _{i} \frac{1-\operatorname{mult}_{E_{i}}(B)}{m_{i}} \leqslant \frac{1}{m_{i}} .
$$

Therefore the fiber $f^{*} P$ is reduced. In particular, there exists a big open subset $Y^{0} \subset Y$ such that $B$ is horizontal over $Y^{0}$ and $f:\left.X\right|_{Y^{0}} \rightarrow Y^{0}$ is smooth on a big open subset of $\left.X\right|_{Y^{0}}$.

(2) Since $\mathbf{M} \sim_{\mathbb{Q}} 0$, there exist by Theorem 3.3 a generically finite morphism $\tau: W \rightarrow Y$ from a nonsingular proper variety $W$ and a nonempty open subset $U \subseteq W$ such that $(X, B) \times\left._{Y} W\right|_{U}$ and $\left(F, B_{F}\right) \times\left. W\right|_{U}$ are isomorphic over $U$. We may assume that the field extension $k(W) / k(Y)$ is Galois and $G=\operatorname{Gal}(k(W) / k(Y))$ acts regularly on $W$. After possibly shrinking $Y^{0}$, we may assume that $W^{0}:=\tau^{-1}\left(Y^{0}\right) \rightarrow Y^{0}$ is a finite, flat Galois covering. Let $X^{\prime} \rightarrow X \times_{Y} W$ be the normalization morphism:

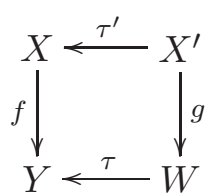

We claim that $X^{\prime} \rightarrow X \times{ }_{Y} W$ is an isomorphism above $W^{0}$. Indeed, restricted to $Y^{0}, f$ is smooth on a big open subset and $\tau^{\prime}$ is finite, hence $X \times\left._{Y} W\right|_{W^{0}} \rightarrow W^{0}$ is smooth on a big open subset of $X \times\left._{Y} W\right|_{W^{0}}$. Since $W$ is nonsingular, the singular locus of $X \times\left._{Y} W\right|_{W^{0}}$ has codimension at least 2. Furthermore, $X \times\left._{Y} W\right|_{W^{0}}$ is $S_{2}$ since $X \times\left.\left._{Y} W\right|_{W^{0}} \rightarrow X\right|_{Y^{0}}$ is finite and flat, and $X$ is $S_{2}$ (see [Mat80, 21.B Theorem 50]).

(3) By finite base change, $f:(X, B) \rightarrow Y$ induces an lc-trivial fibration $g:\left(X^{\prime}, B_{X^{\prime}}\right) \rightarrow W$, with discriminant $\mathbf{B}_{W}$ and moduli b-divisor $\mathbf{M}^{\prime} \sim_{\mathbb{Q}} \tau^{*} \mathbf{M} \sim_{\mathbb{Q}} 0$. Set $B^{\prime}:=B_{X^{\prime}}-g^{*}\left(\mathbf{B}_{W}\right)$. From above, we obtain

$$
g\left(\operatorname{Supp}\left(B^{\prime}\right)\right) \cap W^{0}=\emptyset \quad \text { and } \quad \mathbf{B}_{W}^{\prime}=0 .
$$

The lc-trivial fibration $g:\left(X^{\prime}, B^{\prime}\right) \rightarrow W$ satisfies the assumptions of Proposition 4.4, which gives an isomorphism over $W^{0}$ :

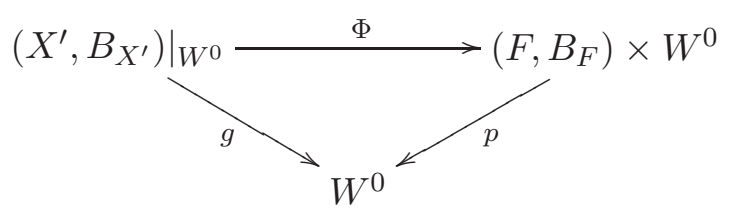

The Galois group $G=\operatorname{Gal}\left(W^{0} / Y^{0}\right)$ acts regularly on $W^{0}$ and $\left.X^{\prime}\right|_{W^{0}}=\left.X\right|_{Y^{0}} \times_{Y^{0}} W^{0}$, and $g$ is $G$-invariant. We have an induced action of $G$ on $\left(F, B_{F}\right) \times W^{0}$ so that $\Phi$ is a $G$-invariant isomorphism.

(4) Let $H$ be the subgroup of $G$ generated by the ramification groups $I(P)$, for every prime divisor $P$ of $W^{0}$. Then

$$
\sigma^{\prime}=\operatorname{id}_{F} \times \sigma \quad \text { for } \quad \sigma \in H,
$$

where $\sigma^{\prime}$ is the automorphism of $\left(F, B_{F}\right) \times W^{0}$ induced by $\sigma$. Indeed, $\sigma^{\prime} \circ\left(\operatorname{id}_{F} \times \sigma\right)^{-1}$ is an automorphism of $\left(F, B_{F}\right) \times W^{0}$ over $W^{0}$, inducing a morphism $s_{\sigma}: W^{0} \rightarrow \operatorname{Aut}\left(F, B_{F}\right)$. For $\sigma \in I(P)$, we have $s_{\sigma}(P)=\left\{\operatorname{id}_{F}\right\}$. Therefore $s_{\sigma}$ maps $W^{0}$ into the connected component of the identity $\operatorname{Aut}^{0}\left(F, B_{F}\right)$. 
We have $s_{\sigma}(w) \cdot \varphi\left(x \times_{Y} \sigma^{-1} w\right)=\varphi\left(x \times_{Y} w\right)$, where $\Phi\left(x \times_{Y} w\right)=\left(\varphi\left(x \times_{Y} w\right), w\right)$. The sections $s_{\sigma}$ satisfy the identity

$$
s_{\sigma \eta}(w)=s_{\sigma}(w) \circ s_{\eta}\left(\sigma^{-1} w\right), \quad \text { for } \quad \sigma, \eta \in H, w \in W^{0} .
$$

Therefore they define a 1-cocycle $\xi=\left\{s_{\sigma}\right\}_{\sigma \in H} \in H^{1}\left(H, A\left(W^{0}\right)\right)$, where $A\left(W^{0}\right)$ is the group of sections of $\operatorname{Aut}^{0}\left(F, B_{F}\right) \times W^{0}$ over $W^{0}$. The $H$-module $\operatorname{Aut}^{0}\left(F, B_{F}\right)$ is commutative by Proposition 4.6 , hence $\xi$ has finite order by [Ser95]. After possibly changing the trivialization, $s_{\sigma}$ is trivial for every $\sigma \in H$.

(5) We have a base change diagram

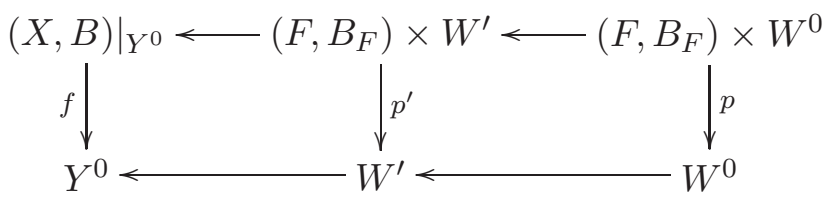

where $W^{\prime}=W^{0} / H$. The covering $W^{\prime} \rightarrow Y^{0}$ is étale Galois and $\operatorname{Gal}\left(W^{\prime} / Y^{0}\right)$ acts on $W^{\prime}$ and $\left(F, B_{F}\right) \times W^{\prime}$ without fixed points.

The normalization of $Y$ in the field $k\left(W^{\prime}\right)$ satisfies the required properties.

Theorem 4.8. Let $(X, B)$ be a projective $\log$ variety with Kawamata log terminal singularities such that $K+B \sim_{\mathbb{Q}} 0$. Then:

(i) the Albanese map $X \rightarrow \operatorname{Alb}(X)$ is a surjective morphism, with connected fibers;

(ii) there exist a finite étale covering $A^{\prime} \rightarrow \operatorname{Alb}(X)$, a projective $\log$ variety $\left(F, B_{F}\right)$, and an isomorphism over $A^{\prime}$ such that

$$
\left(F, B_{F}\right) \times A^{\prime} \stackrel{\sim}{\longrightarrow}(X, B) \times \operatorname{Alb}(X) A^{\prime} .
$$

Proof. (i) Let $X \stackrel{f}{\rightarrow} Y \stackrel{\pi}{\rightarrow} A=\operatorname{Alb}(X)$ be the Stein factorization of $\alpha_{X}$. Since $f$ is a contraction and $K+B \sim_{\mathbb{Q}} 0, f:(X, B) \rightarrow Y$ is an lc-trivial fibration with

$$
K_{Y}+\mathbf{B}_{Y}+\mathbf{M}_{Y} \sim_{\mathbb{Q}} 0 .
$$

Since $\pi$ is finite on its image, any resolution of $Y$ has nonnegative Kodaira dimension [Uen75, Theorem 10.3]. Therefore $K_{Y}$ is $\mathbb{Q}$-linearly equivalent to an effective $\mathbb{Q}$-Weil divisor. Moreover, $\mathbf{B}_{Y}$ is effective since $B$ is effective, and $\mathbf{M}_{Y}$ is $\mathbb{Q}$-linearly equivalent to an effective $\mathbb{Q}$-Weil divisor since $\kappa(\mathbf{M}) \geqslant 0$. Therefore $K_{Y} \sim_{\mathbb{Q}} 0, \mathbf{B}_{Y}=0$ and $\mathbf{M}_{Y} \sim_{\mathbb{Q}} 0$. The latter implies $\mathbf{M} \sim_{\mathbb{Q}} 0$, since $\mathbf{M}$ is b-nef and good.

By inversion of adjunction, $Y$ has Kawamata log terminal singularities and $K_{Y} \sim_{\mathbb{Q}} 0$. The index one cover $\tilde{Y} \rightarrow Y$ has canonical singularities and $K_{\tilde{Y}} \sim 0$. Therefore $\tilde{Y}$, hence $Y$, map onto $A$ by [Uen75, Theorem 10.3]. The finite map $\pi: Y \rightarrow A$ is étale in codimension 1 since $0 \leqslant R=K_{Y}-\tau^{*}\left(K_{A}\right) \sim_{\mathbb{Q}} 0$. Since $A$ is nonsingular, $\pi$ is étale everywhere. In particular, $Y$ is an Abelian variety. Therefore $\pi$ is an isomorphism, by the universality of the Albanese map.

(ii) We have an lc-trivial fibration $\alpha_{X}:(X, B) \rightarrow A$ with $\mathbf{B}_{A}=0$ and $\mathbf{M} \sim_{\mathbb{Q}} 0$. By Theorem 4.7, there exists a finite morphism $\tau: A^{\prime} \rightarrow A$, étale in codimension 1, from a normal variety $A^{\prime}$ such that $(X, B) \times{ }_{A} A^{\prime}$ is isomorphic to $\left(F, B_{F}\right) \times A^{\prime}$ over the generic point of $A^{\prime}$. Since $A$ is nonsingular, $\tau$ is étale everywhere. Since $\mathbf{B}_{A}=0$ and $A^{\prime}$ is nonsingular, we get an isomorphism $(X, B) \times{ }_{A} A^{\prime} \rightarrow$ $\left(F, B_{F}\right) \times A^{\prime}$ by Proposition 4.4 . 


\section{F. AmBro}

\section{ACKNOWLEDGEMENTS}

I would like to thank Professors Alessio Corti, Nick I. Shepherd-Barron and Vyacheslav V. Shokurov for useful discussions. This work was supported through a European Community Marie Curie Fellowship.

\section{REFERENCES}

Amb99 F. Ambro, The Adjunction Conjecture and its applications, Preprint (1999), math.AG/9903060.

Amb02 F. Ambro, Shokurov's boundary property, J. Differential Geom. 67 (2004), 1-27.

Amb03 F. Ambro, Nef dimension of minimal models, Math. Ann. 330 (2004), 309-322.

CPZ03 F. Campana, T. Peternell and Q. Zhang, On the Albanese maps of compact Kähler manifolds, Proc. Amer. Math. Soc. 131 (2003), 549-553.

DPS93 J.-P. Demailly, T. Peternell and M. Schneider, Kähler manifolds with numerically effective Ricci class, Compositio Math. 89 (1993), 217-240.

FM00 O. Fujino and S. Mori, A canonical bundle formula, J. Differential Geom. 56 (2000), 167-188.

Fuj78a T. Fujita, On Kähler fiber spaces over curves, J. Math. Soc. Japan 30 (1978), 779-794.

Fuj78b T. Fujita, The sheaf of relative canonical forms of a Kähler fiber space over a curve, Proc. Japan Acad. Ser. A Math. Sci. 54 (1978), 183-184.

Fuj86 T. Fujita, Zariski decomposition and canonical rings of elliptic threefolds, J. Math. Soc. Japan 38 (1986), 19-37.

Fuj03 O. Fujino, A canonical bundle formula for certain algebraic fiber spaces and its applications, Nagoya Math. J. 172 (2003), 129-171.

GK64 H. Grauert and H. Kerner, Deformationen von Singularitäten komplexer Räume, Math. Ann. 153 (1964), 236-260.

Gra74 H. Grauert, Der Satz von Kuranishi für kompakte komplexe Räume, Invent. Math. 25 (1974), 107-142.

Gri70 P. A. Griffiths, Periods of integrals on algebraic manifolds. III. Some global differential-geometric properties of the period mapping. Publ. Math. Inst. Hautes Études Sci. 38 (1970), 125-180.

Gro62 A. Grothendieck, Fondaments de la géométrie algébrique: extraits du Séminaire Bourbaki, 1957-1962 (Institut Henri Poincaré, Paris, 1962).

Hir64 H. Hironaka, Resolution of singularities of an algebraic variety over a field of characteristic zero: I, Ann. of Math. (2) 79 (1964), 109-203.

Hir77 H. Hironaka, Bimeromorphic smoothing of a complex-analytic space, Acta Math. Vietnam. 2 (1977), 103-168.

Kaw78 Y. Kawamata, On deformations of compactifiable complex manifolds, Math. Ann. 235 (1978), $247-265$.

Kaw81 Y. Kawamata, Characterization of abelian varieties, Compositio Math. 43 (1981), 253-276.

Kaw83 Y. Kawamata, Kodaira dimension of certain algebraic fiber spaces, J. Fac. Sci. Univ. Tokyo 30 (1983), 1-23.

Kaw85 Y. Kawamata, Minimal models and the Kodaira dimension of algebraic fibre spaces, J. reine angew. Math. 363 (1985), 1-46.

Kaw97 Y. Kawamata, Subadjunction of log canonical divisors for a variety of codimension 2, Contemp. Math. 207 (1997), 79-88.

Kaw98 Y. Kawamata, Subadjunction of log canonical divisors, II, Amer. J. Math. 120 (1998), 893-899.

Kol87 J. Kollár, Subadditivity of the Kodaira dimension: fibers of general type, in Algebraic geometry (Sendai, 1985), Advanced Studies in Pure Mathematics, vol. 10 (North-Holland, Amsterdam, 1987), 361-398.

Mat80 H. Matsumura, Commutative algebra, second edition, Mathematics Lecture Note Series, vol. 56 (Benjamin/Cummings, Reading, MA, 1980). 


\section{THE MODULI B-DIVISOR}

MO67 H. Matsumura and F. Oort, Representability of group functors and automorphisms of algebraic schemes, Invent. Math. 4 (1967), 1-25.

Nak88 N. Nakayama, On Weierstrass models, in Algebraic geometry and commutative algebra, vol. II (Kinokuniya, Tokyo, 1988), 405-431.

PS98 T. Peternell and F. Serrano, Threefolds with nef anticanonical bundles. Dedicated to the memory of Fernando Serrano, Collect. Math. 49 (1998), 465-517.

Ran89 Z. Ran, Deformations of maps, in Algebraic curves and projective geometry (Trento, 1988), Lecture Notes in Mathematics, vol. 1389 (Springer, Berlin, 1989), 246-253.

Ros56 M. Rosenlicht, Some basic theorems on algebraic groups, Amer. J. Math. 78 (1956), 401-443.

Ser95 J.-P. Serre, Espaces fibrés algébriques, Séminaire Bourbaki, vol. 2, exp. no. 82 (Soc. Math. France, Paris, 1995), 305-311.

Sho03 V. V. Shokurov, Prelimiting flips, in Birational geometry: linear systems and finitely generated algebras, eds V. A. Iskovskikh and V. V. Shokurov, Proc. Steklov Inst. Math. 240 (2003), 82-219.

Ste77 J. H. M. Steenbrink, Mixed Hodge structure on the vanishing cohomology, in Real and complex singularities (Proc. Ninth Nordic Summer School/NAVF Sympos. Math., Oslo, 1976) (Sijthoff and Noordhoff, Alphen aan den Rijn, 1977), 525-563.

Uen75 K. Ueno, Classification theory of algebraic varieties and compact complex spaces, Lecture Notes in Mathematics, vol. 439 (Springer, Berlin, 1975).

Vie83a E. Viehweg, Weak positivity and the additivity of the Kodaira dimension for certain fibre spaces, in Algebraic varieties and analytic varieties (Tokyo, 1981), Advanced Studies in Pure Mathematics, vol. 1 (North-Holland, Amsterdam, 1983), 329-353.

Vie83b E. Viehweg, Weak positivity and the additivity of the Kodaira. II: The local Torelli map, Progress in Mathematics, vol. 39 (Birkhäuser, Boston, MA, 1983), 567-589.

Zha96 Q. Zhang, On projective manifolds with nef anticanonical bundles, J. reine angew. Math. 478 (1996), $57-60$.

Florin Ambro ambro@kurims.kyoto-u.ac.jp

RIMS, Kyoto University, Kyoto 606-8502, Japan 